(9) When rennet enzyme was the only digesting agent in cheese. we were unable, in any case, to find the slightest traces of cheese flavor. Apparently, we must look to other sources for this : $m$ portant product of cheese-ripening.

New York Agricultural Experimest Station, Geneva N. Y.

\title{
A METHOD OF GRADING SOAPS AS TO THEIR DETER- GENT POWER.
}

BY H. W. HILIYER.

Received October $7 \cdot 1903$.

As FAR as known to the writer, no method for directly determining the detergent value of a soap is now known. It is assumed, and with much justice, that the value of a soap is a function of the amount of combined fatty acids present, and the valuation of soaps is now based on the determination of the fatty acids. Besides this, the determination of the free alkali and of water and various filling materials is required. These determinations give evidence on which the valuation of the soap may be based and, to some extent, for ascertaining the materials from which it is made, but do not give any direct measure of the value as a cleansing, agent and, further, require a complex judgment to interpret them.

The method now proposed is based on the study of sodium soaps, previously publishd in this Journal, 25, $5 \mathrm{II}$ and 524 . In the articles indicated, it was found that when a solution of a soap is made to form drops beneath the surface of an oil, the number of drops formed from a given volume of the solution is dependent on the amount of soap present in the solution. Further, the conclusion was reached that the number of drops formed was a measure of the emulsifying power of the given soap solution, and that the emulsifying power was so large a part of the cleansing power that it might stand as a measure of it, especially, since two of the other probable factors in cleansing, namely. penetrating or wetting power, and lubricating power are dependent on the same physical properties as the emulsifying power. 
Soaps are used principally in one of two ways. They are used with cold or luke-warm water, as in wool-scouring and for toilet purposes; or, on the other hand, they are used in boiling water, as in silk-boiling, dish-washing, and laundry work. A soap may be of value for one of these purposes and of comparatively little value for the other. The method proposed takes these two peculiar conditions of use into account and attempts to grade the soaps as to their efficiency for cold use and, on the other hand, for use with hot water.

For making the test for efficiency in use with hot water, the apparatus is similar to that used for the study of sodium palmitate and stearate, but modified in the interest of greater constancy of results. The essential part is a pipette (A) with a capillary outlet of about $0.5 \mathrm{~mm}$. internal diameter and a flat, horizontal surface, about $10 \mathrm{~mm}$. in diameter, from which the drops fall. This surface must be free from capillary openings, which may contain air, as described in a former article. The reservoir of the pipette is spherical, that it may have a small vertical height relatively to its volume, since a great vertical height makes a greater difference in the pressure and consequent rate of flow at the commencement and at the end of an observation. When the initial pressure is great, the rapidity of flow may make the solution run down in a stream or cause the drops to be formed too rapidly to be easily counted. This may be overcome by dipping the pipette more deeply into the oil; but when that is done the later drops are formed more slowly than is desirable. By having the bulb capacious in proportion to its vertical height the initial and final rates of flow are nearly enough alike to prevent any special difficulty. The pipette holds 5 cc. from a mark on the stem to the upper end of the capillary. The stem has an internal diameter of $3 \mathrm{~mm}$., wide enough to allow filling from above, and, to facilitate this method of filling, it is widened at the upper end into a small funnel.

The second part of the apparatus is the receptacle for the oil, which may be a simple test-tube, but is preferably made in the form $(\mathrm{BC})$ indicated in the drawing. This form maintains a more nearly constant pressure and promotes more regular work. To maintain the apparatus at the boiling-point of water, it is supported in a large beaker, which is used as a water-bath. The 
beaker is covered with a sheet of zinc or aluminum with its edges turned down over the edge of the beaker, and with a perforation cut in it suitable for the insertion of the apparatus.

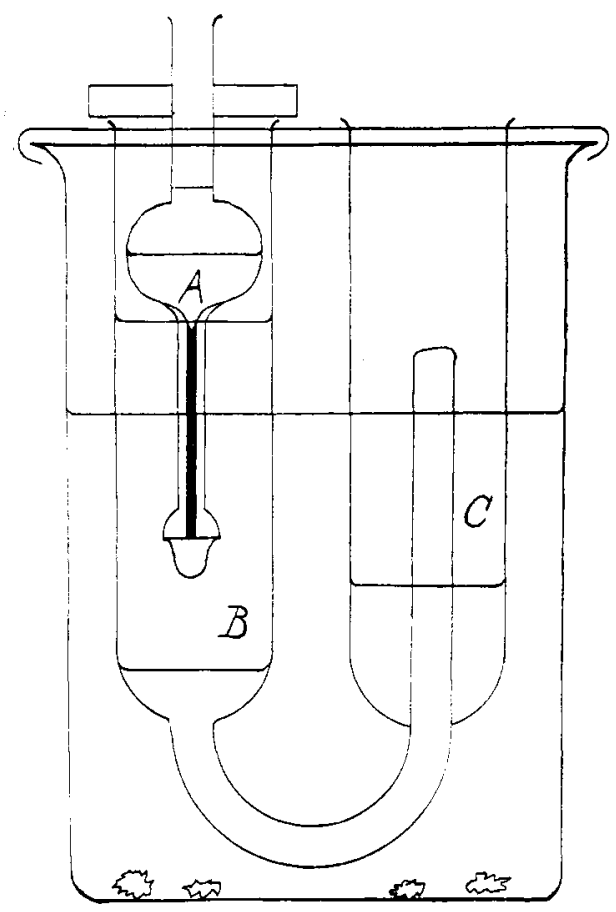

To make use of the apparatus, 2 or $3 \mathrm{cc}$. of water are poured into $B$ and then followed by $20 \mathrm{cc}$. of kerosene. A little of the solution to be tested, previously heated on a boiling waterbath, is poured into the pipette and shaken about, and then blown out through the capillary, using a piece of rubber tubing as a mouth-piece. This is repeated twice more. Then the pipette is filled full, and by a strong pressure from the lungs the air bubbles are forced out of the capillary and washed away. The pipette, supported in a cork, is placed in the kerosene, and the number of drops formed from $5 \mathrm{cc}$. of the solution is counted. The solution, as it flows from the pipette, falls to the bottom of the oil reservoir $B$ and overflows into $C$, while the level of the kerosene remains constant and the variation of pressure is that only which results from the change of level of the solution in the pipette. 
Since this is in every case the same, comparable results are more easily obtained than by the use of a test-tube as oil reservoir.

To make a test of efficiency of the soap for use in cold water, the same apparatus is used, except that the beaker, used as a water-jacket, is not necessary.

As a standard for the hot test, I gram of neutral sodium palmitatc, dissolved in zco cc. of hot water, is used. Of this, Io cc., $20 \mathrm{cc}$, $30 \mathrm{cc}$, and $40 \mathrm{cc}$. are, respectively, diluted to $50 \mathrm{cc}$. with boiling water, giving, solutions containing $0.2,0.4,0.6$, and 0.8 of the standard. The number of drops formed by each of these solutions, when it flows into a kerosene, adopted as a standard kerosene, is determined, and also the number of drops formed by the standard solution itself and the number of drops formed by water. The data obtained are plotted as a curve in which the ordinates represent percentages of the standard and the abscissas represent the number of drops corresponding to the percentages of the standard. The data, obtained with a certain kerosene, are here given and the curve $(A)$ derived is shown in the figure.

The standard is made by bringing together $0.92 \mathrm{I}$ gram of palmitic acid and $36 \mathrm{cc}$. decinormal sodium hydroxide and diluting to $200 \mathrm{cc}$. with hot water.

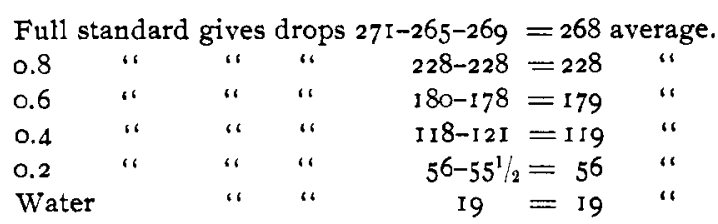

If any solution of sodium palmitate, which contains an amount less than one part in 200 of water, is tested by the dropping method at the boiling-point, the amount present may be determined by counting the number of drops formed. By finding, on the curve, a point corresponding to the number of drops counted and letting fall a perpendicular from this point upon the base line, a point will be found which indicates, as a percentage of the standard, the amount of palmitate present.

Other soaps may be tested in the same way and referred to sodium palmitate as a standard. If $0.5 \mathrm{gram}$ of a commercial soap is dissolved in $100 \mathrm{cc}$. hot water and its solution tested at the boiling-point, we may state the result in this way: The soap 


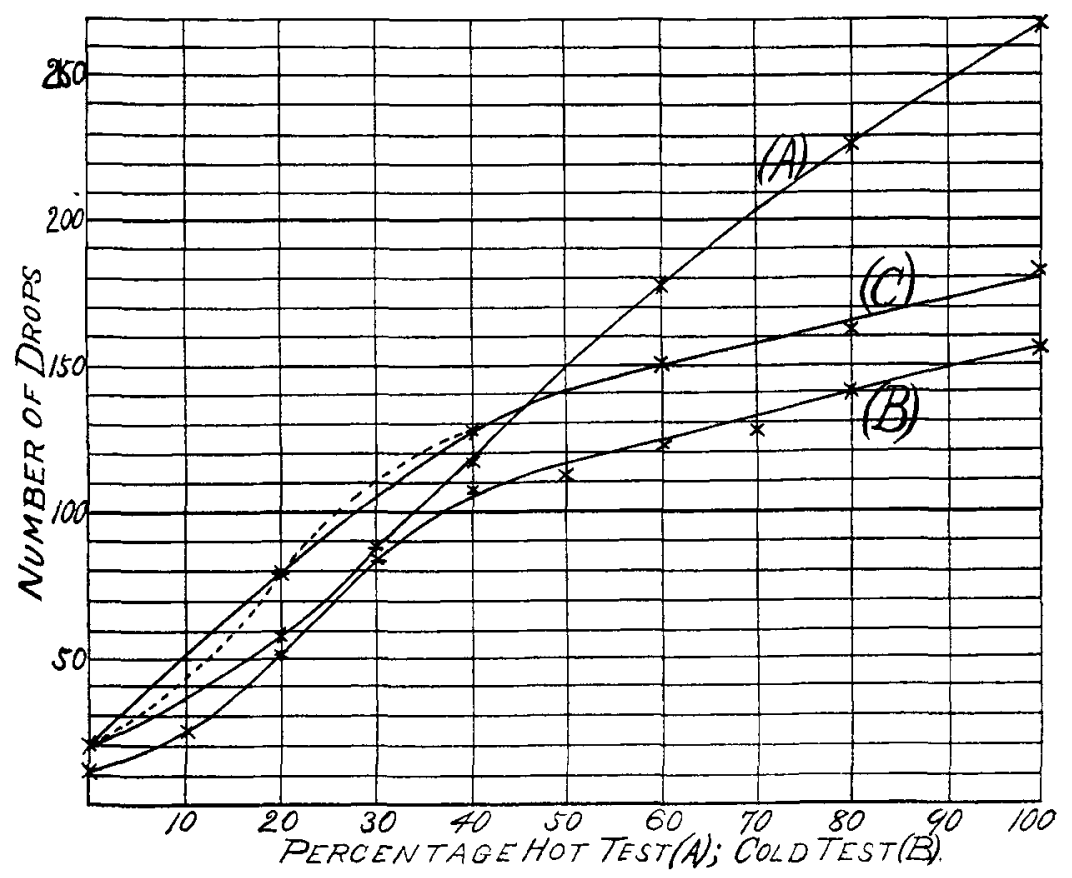

in question gives the number of drops which would be given by a solution of sodium palmitate containing ( $x$ ) per cent. of the standard.

According to the conclusions arrived at in the previous articles, the number of drops is a measure of the cletergent power of the solution. Consequently, the result of the test may be stated as follows: The soap has, when used for cleansing with hot water, a detergent power as great as a soap containing $(x)$ per cent. of sodium palmitate.

TEST FOR COLD-WATER SOAPS.

A solution containing one part of sodium oleate in 200 parts of cold, freshly boiled, distilled water is taken as the Ioo per cent. standard for soaps to be used with cold water. From this, fractional standards are made as before, using cold, freshly boiled, distilled water to dilute with. All are tested as to their drop number in the cold and a curve constructed. In a previous article it was stated that the curve for sodium oleate was differ- 
ent in shape from the curves for the other sorium soaps tested. The following data gives a curve for oleate (B), which appears to have nearly the same form as the curves previously given for rosin soap and different from the curves formerly given for oleate. But this difference is due to the greater dilution of the solution and to the greater number of fractional standards, and to the fact that observations are made nearer the origin. The curve shows that sodium oleate is somewhat hydrolyzed at great dilutions. A set of standards gave the following data and curve (B).

\begin{tabular}{|c|c|c|c|c|c|}
\hline Fu & & & & $53=$ & \\
\hline 0.8 & " & "“ & " & $\mathrm{I} 39-\mathrm{I} 4 \mathrm{I}=\mathrm{I} 40$ & “" \\
\hline 0.7 & “ & "“ & "6 & $128-128=128$ & \\
\hline 0.6 & “" & “" & " & $123-\mathrm{I} 22=\mathrm{I} 23$ & \\
\hline 0.5 & "“ & " & “ & $\operatorname{III-III}=\operatorname{III}$ & \\
\hline 0.4 & “ & “ & " & $107-105=106$ & \\
\hline 0.3 & “" & " & " & $84=84$ & \\
\hline 0.2 & “" & " & $"$ & $5^{I-50}=5 \mathrm{I}$ & \\
\hline 0.1 & " & " & ‘ & $25-25=25$ & \\
\hline Wa & & "“ & "4 & $I I-I I=I I$ & \\
\hline
\end{tabular}

For evaluating a commercial soap for efficiency in use with cold water, the following method of working was decided upon after trying several others, which will be indicated later. The soap to be tested is shaved as thin as possible, and 0.5 gram of the shavings weighed and placed in a IOO cc. flask. The flask is then filled to the mark with cold, freshly boiled water and allowed to stand over night. In the morning the solution is stirred, and filtered through a dry filter, letting all run through that will and keeping the solution in the funnel protected from the carbon dioxide of the air by a watch-glass. When all has run through, the filtrate is stirred gently, avoiding making a foam, and a dropping test in the cold is made, using the same pipette and kerosene as that used in testing the standards. By applying, to the standard curve, the drop number observed, a number is obtained which may be stated, as in case of the hot test. A certain number of drops shows that the soap in question is as efficient for cold cleansing as an equal amount of soap containing $(x)$ per cent. of sodium cleate.

Since surface-tension, viscosity, specific gravity and solubility, factors which enter more or less into the working of the test, are all influenced by changes of temperature, it is best to maintain 
the temperature constant, as nearly as possible, in all the work involved in making the cold test. It will be convenient, in many cases, to select a convenient room temperature and maintain this, as nearly as possible, during the preparation and testing of the standards, and the preparation and testing of the soap solutions to be evaluated. If many tests are to be made, a trough with glass sides, through which a stream of hydrant water is maintained, might be convenient to set the flasks in and to receive the oil-holder while observations are being made.

Data obtained with a few commercial soaps are here given, showing for each the number of drops formed in the cold test and the percentage efficiency for cold use, indicated by the test; and the drop number by the hot test and percentage efficiency for hot use. The soaps tested are nearly all much-advertised soaps.

\begin{tabular}{|c|c|c|c|c|c|}
\hline \multirow[b]{2}{*}{ Number. } & \multicolumn{2}{|c|}{ Cold. } & \multicolumn{2}{|c|}{ EIot. } & \multirow{3}{*}{$\begin{array}{l}\text { Remarks. } \\
\text { Cold made, cocoanut oil. }\end{array}$} \\
\hline & Drops. & Efficiency, & Drops. & Efficiency. & \\
\hline 1 & I 2 I & 37 & 90 & $3 \mathrm{I}$ & \\
\hline 2 & 56 & $\mathrm{I}_{3}$ & 67 & $2 \mathrm{I}$ & Hygienic cocoanut oil. \\
\hline 3 & I I 4 & 34 & 221 & 77 & Toilet. \\
\hline 4 & $\operatorname{lig}$ & 36 & 202 & 69 & Shaving. \\
\hline 5 & 84 & 22 & 80 & 28 & Tar, toilet. \\
\hline 6 & 75 & I9 & 103 & 35 & Yellow laundry, rosin. \\
\hline 7 & 100 & 28 & 135 & 4.5 & " \\
\hline 8 & 107 & $3 \mathrm{I}$ & 173 & 58 & Household for cold use. \\
\hline 9 & 98 & 27 & 150 & 50 & " \\
\hline IO & I 39 & 49 & 170 & 57 & " $\quad$ " $\quad$ ، \\
\hline II & 97 & 27 & 206 & 71 & White laundry and toilet. \\
\hline I 2 & $\ldots$ & $\ldots$ & 226 & 79 & " $\quad$ " \\
\hline I 3 & $\ldots$ & . & 216 & 75 & Prime yellow laundry. \\
\hline I4 & I 20 & 37 & $2 \mathrm{is}$ & 76 & " $"$ " \\
\hline I5 & JO4 & 30 & 277 & 106 & Steam laundry, tallow. \\
\hline I6 & $I_{42}$ & $5: 4$ & $\cdots$ & $\cdots$ & Castile, old and dry. \\
\hline
\end{tabular}

These results show how wide a ditference there is between soaps which have wide sale and also the desirability of having some standard by which to readily judge them. In the retail market, many household soaps sell at the price of five cents per bar. The bars vary very much in weight, but it was found that in a number of cases the value per bar was approximately equal according to the dropping test. For example, No. 6 is sold in bars weighing 330 grams; its hot test shows 35 per cent. The product of these, or efficiency per bar is I 5.5 . No. 7 is sold in 
270-gram bars. The hot test shows 45 per cent., or efficiency per bar, I 21.5 . No. I I is sold in I60-gram bars. Hot test, 7 I per cent. Efficiency per bar, I I3.6.

This calculation shows that in some cases the manufacturers have correctly estimated the relative value of their product.

From the table it may be seen that those soaps intended for toilet purposes or advertised for cold use are, for the most part, of a relatively high efficiency by the cold test. No. I6 is a dry, old, standard castile soap and shows the highest cold test of any. But it is somewhat surprising that even this is only a little more than half as efficient as pure sodium oleate.

No. I5 is a soap known to be made from tallow. It is consumed by local steam laundries and regarded by them as the best soap they can find for their purpose. According to the hot test, it is above the standard palmitate in efficiency. Nos. I I, I2, I3, and 14 are among the most highly appreciated family soaps on the market, and show a high hot test.

That there is not a complete agreement between the tests and the advertised uses is not strong evidence against the test. The makers may be mistaken in their judgment of the best way to use their soaps, or they may, in some cases, purposely advertise them for uses to which they are not well adapted.

It is not expected that the method, at least in its present form, will give a very exact measure of the value of soaps, but it is hoped that, in a field where there is no exact method, the one here proposed may aid consumers in selecting soaps fitted to their special needs and in writing specifications by which soaps may be contracted for. It is also hoped that it may lead to a more concordant judgment, on the part of manufacturers, as to what a normal soap is, and as to how far it is legitimate to vary from this normal soap. If some ready method of judging a soap is adopted, it may seem unprofitable both to maker and consumer to pay freight charges on water and fillers which are of no real detergent value.

The hot test may be relied upon to give results concordant within I per cent. for successive samples and within a much closer limit in duplicate tests of the same sample. The cold test is not as accurate as the hot test on account of the colloidal 
nature of the solution. It will probably grade soaps within 4 or 5 per cent. in the case of soaps whose solutions are very slimy, and within a closer limit in cases in which the solutions are mobile.

The working out of the hot test presented no particular difficulty, since the soaps worked with dissolve in hot water to give mobile solutions which readily flow through the pipette and can be readily duplicated. In working out the cold test, the method indicated was adopted only when other methods proved unsatisfactory. It is thought best to give an account of some of these attempts that others may not find it necessary to repeat them without modification.

At first the solutions, prepared for the hot test, were allowed to cool, then poured upon a filter and the filtrate tested cold. But the first portion of the filtrate gives a lower drop number than the later portions, and even if all is allowed to drain off before testing, and in this way an average for the whole sample obtained, the drop numbers for successive samples is not at all concordant. These difficulties are probably due to the great variation in the solubility of the colloidal precipitate which separates, with small differences in temperature and to the slowness with which equilibrium is reached between the dissolved and undissolved colloid.

It was thought that by making the solutions more dilute, they might have a low enough viscosity, when cold, to flow through the pipette without filtering. This was found to be the case when the solutions contained only one part in 400 parts of water but on testing several soaps of different classes in this way, they all showed nearly the same cold test and no comparison could be based on the results.

Another attempt was made by adding a solution of common salt to the soap solution and then filtering. It was found that when equal quantities of 2.5 per cent. sodium chloride solution were added to 0.5 per cent. sodium palmitate solution, that nearly all of the palmitate was precipitated and the filtrate showed only a small drop number. When the same salt solution was added to 0.5 per cent. oleate solution there was, at first, no precipitation and the drop number was increased. But the oleate solution with salt, on making successive tests, showed a rapidly decreasing drop number, making it impossible to use this mixture as a basis 
for a standard curve. The decrease in number of drops is very soon apparent, the solution becomes cloudy, and, in a few days, nearly all of the oleate appears to be precipitated, while the filtrates, no matter what the original strength, show a strong tendency to the same minimum drop number.

IABORATORY OF ORGANIC CHEMISTRY,

UNIVERSITY OF WISCONSIN, June, rgo3.

\section{A RAPID METHOD FOR THE DETERMINATION OF SUL- PHUR IN COAL AND COKE.}

BY J. D. PENNoCk AND D. A. MORTON,

Received October 5,1903 .

As is well known, foundrymen require coke containing a minimum percentage of sulphur. It has been found necessary to be able to quickly determine the percentage of sulphur in the coal to be used for coke-making in order that a selection of those cars containing coal low in sulphur, may be made, for the railroad companies chrarge demurrage on all cars not emptied in twenty-four hours. By the method described in this paper, one chemist has been able to make twenty determinations of sulphur in six hours.

'The determination of sulphur in coals and cokes, as described in this paper, is, in its essential features, a combination of parts of two methods already in use. These parts are, first, the process used by Sundstrom ${ }^{1}$ for the complete oxidation of coals by means of sodium peroxide; second, the method of Andrews ${ }^{2}$ for the volumetric estimation of the sulphur thus formed.

In carrying out these processes, however, various modifications have been introduced in order to accomplish, as perfectly as possible, the end in view; namely, the accurate, rapid, and convenient estimation of sulphur in coals and cokes. This object is satisfactorily accomplished by following the method as outlined below.

APPARATUS AND SOLUTIONS.

Crucible.-A soft steel crucible of about $40 \mathrm{cc}$. capacity, the lid being perforated with a small hole for the introduction of the igniting wire.

1 This Journal, February, 1903.

${ }^{2}$ Am. Chem. J., 11, 567;J. Anal. Chem., 4, 73 (1890). 